\title{
Dissolution profiling and its comparison of natural fruit powder effervescent tablets
}

\begin{abstract}
The aims of the study were to observe the dissolution profile of four types of selected fruit powder fast dissolve tablet and apply different dissolution profile comparison methods, in order to select most applicable method. Spray dried powder of pitaya, pineapple, guava and mango were used as raw material. Each type of fruit powder tablet has identical dissolution profile. Dissolution profiles and dissolution rate of fruit powder tablets were different at different dissolution environment. In model-independent and statistical method pairwise comparison was performed and in model dependent method profile comparison was carried out based on best fitting of mathematical model equation. Dissolution Profile comparison methods represented that model independent method use wide range of value for profile comparison and it was direct and easy; model dependent method was indirect and complex and for critically compare the dissolution profile statistical method was the most suitable method.
\end{abstract}

Keyword: Fruit powder tablet; Dissolution profiling; Profile comparison; Model dependent method; Model independent method; Statistical method 